\title{
Effects of Background Context for Objects in Photographic Targets on Remote Viewing Performance
}

\author{
Debra Lynne Katz \\ International Remote Viewing Association, USA \\ James D. LANe \\ Rhine Research Center, Durham, North Carolina, USA \\ Michelle Freed-Bulgatz
}

Submitted July 11, 2020; Accepted August 8, 2021; Published December 30, 2021

https://doi.org/10.31275/20212273

Creative Commons License CC-BY-NC

Abstract-Photographic images of physical objects are common targets in remote viewing projects today. This exploratory experiment investigated whether the background within which the object is positioned may impact the accuracy of remote viewing. Twelve experienced remote viewers each completed 30 open-response, triple-blind remote viewing trials, requiring them to utilize extrasensory perception to describe the photographic image they would receive via email a few days later. Investigators created a photographic target pool of complex objects set within one of three background conditions: 1) White: devoid of information; 2) Normal: a setting in which the object would typically be found; 3) Unusual: a setting in which the object would not typically be found. Participants completed a total of 360 in-depth transcripts consisting of 8,460 written descriptors and 1,472 sketches. Two methods were used to analyze the transcripts for accuracy, the traditional sum of ranks matching procedure and an exploratory method involving the scoring of each item and sketch by both the participant and an independent judge. These two methods revealed significant but opposite differences for photographic targets of objects set within white backgrounds compared to the other two backgrounds. Better scores for targets with a white background were found for the traditional matching procedure, but worse scores were found for this background when each item and sketch were rated individually. In 
addition, the individual items and sketches were found to describe the target object more frequently than the background when normal or unusual backgrounds were present. Results suggest that object background can affect the outcome of remote viewing sessions, although the effect may depend on the scoring method applied.

Keywords: Remote viewing, target material, extrasensory perception, anomalous cognition, photographic target material

\section{BACKGROUND}

Within the parapsychological literature, an emphasis has been placed on certain target material characteristics that might lead to greater success in free-response experiments involving nonlocal, psibased perception. These characteristics include: familiarity with one's natural environment (Pratt et al., 1940; Sinclair, 1930; Warcollier, 1948); movement-dynamic vs. static (Honorton \& Schechter, 1987; Honorton et al., 1990; Krippner \& Zeichner, 1974; Morris, 1977; Warcollier, 1948); surprising, interesting, and meaningful content but not disturbing (Delanoy, 1989; Nash \& Nash, 1961; Tart, 1980; Warcollier, 1938; Watt, 1989); use of objects or elements in which the foreground is distinctive from the background (Warcollier, 1948; Watt, 1989); and the portrayal of a potentially realistic scene or object vs. one that is abstract or presented in an unrealistic or unrecognizable fashion (Delanoy, 1989; Krippner \& Zeichner, 1974). May (2011) noted that targets with thermodynamic properties, those involving the release of a large amount of energy in a short period of time, such as nuclear tests and rocket launchings, "never seemed to fail" (p. 65). Some researchers found that emotiontriggering images such as those containing sexual content sometimes produced stronger effects under certain conditions (Honorton, 1985; Bem, 2011).

A re-occurring theme across all the above studies was that individual participants often differed in their emotional responses to certain targets. Warcollier $(1938,1948)$ found that those who tended to exhibit stronger emotional reactions than others in their lives seemed to be impacted by the emotionality of targets more than others as well. Delanoy (1989) found what one is normally attracted to in regular 
perception tends to be the same thing that one is attracted to with psi perception, so for example a person who enjoys cityscapes over natural landscapes may have better success at scenes of cities over nature scenes. Additionally, many researchers have suggested that psi perception may parallel regular perception in terms of a participant's ease or difficulty in perceiving different types of targets or aspects of them. Warcollier (1948) argued that certain principles emerging from the Gestalt schools of psychology, such as figure and ground (the ways in which one distinguishes a figure from the background) and closure (the tendency to fill in missing information from an incomplete object) were applicable to emerging data from his own telepathy experiments. He stated, "We can look to the psychology of perception for other principles that reveal themselves in paranormal behavior" ( $p$. 26). Schmeidler (1977) wrote "find what a person perceives best and perceives less accurately, whether in vision, auditory, etc., then test the hypothesis in remote viewing that he will be most accurate and least accurate-in parallel ways" (p. 1). Other researchers who theorized that psi perception may mirror regular perception included Pratt et al. (1940), Mitchell (1981), Watt (1989), May and Lantz (1991), May et al., (1994a, 1994b), Swann and Puthoff (1987), Targ et al. (1995), and Thorpe (2013).

Types of objects used in successful free-response telepathy, clairvoyant, dream ESP, GESP, Ganzfeld, and remote viewing experiments and applied projects spanning the past 100 years have included: simple drawings (Carrington, 1941; Sinclair, 1930; Warcollier, 1948); video clips (Krippner \& Zeichner, 1974; Storm et al., 2010); picture compilations or collages including a mixture of photos and cartoon-like drawings (Honorton, 1985; Krippner et al., 2018); photographs of real locations (Katz et al., 2019a, 2019b; Müller et al., 2019); actual locations (May et al., 1990; Schwartz, 1977, 2019; Targ \& Puthoff, 1977, 2005); and real objects (Mitchell, 1988; Targ \& Puthoff, 1974, 1977; Targ et al., 1995).

\section{Rationale for Current Project}

In recent times, remote viewing projects outside of academic research settings have moved toward the use of photographs of objects as targets, encouraged by the ease of acquisition of photographic images through free or inexpensive online photo-sharing sites. This 
provides a much broader range of potential target objects. In these images the object is sometimes shown on a white or plain-colored background devoid of information, and sometimes it is set within a real background that can both provide contextual information about a target, but that could potentially distract from the main focus.

Despite lack of formal testing of the importance of image format, these varied images have often been used, perhaps forming the entire target pool or mixed with more traditional photographic images of actual locations, in applied and experimental remote viewing projects. Most of these projects have not been reported in the research literature, although the present researchers have participated in them as remote viewers and judges. These have been carried out by parapsychological researchers attempting exploratory studies, and by applied RV or ARV project managers (Katz et al., 2018a, 2018b; Rosenblatt, 2000; Rosenblatt et al., 2015; Williams \& Siegel, 2014) for purposes of using psi for wagering in stock market predictions or sporting events or horse races.

\section{Objects within Normal Settings vs. Unusual Settings}

Additionally, one of the present researchers (the first author) noticed that many of her remote viewing students, who were located at various distances and meeting via teleseminar conferencing, had an easier time recognizing larger gestalts, even naming the target, when real objects were set in normal locations vs. unusual ones. A normal background would be a boat in the water, or a piano located in a living room. Unusual might be a boat or a piano positioned in a desert landscape. This seemed in alignment with results from earlier studies that Delanoy (1989) included in her literature review of target characteristics.

\section{A Theoretical Model for a Conceptual Replication}

We conducted a search of the more recent cognitive attention literature involving types of photographs that are more easily perceived than others. Of greatest relevance was a series of experiments conducted by Barenholtz (2013). The experimenters sought to understand factors involved in visual recognition of objects as they are related to environmental settings. While most visual research had focused on 
the inherent properties of objects, Barenholtz wanted to understand the relationship of visual context to object recognition through testing reaction time. The study involved comparing the time it would take for a participant to recognize an object when it was set within one type of setting or background, compared to another. To measure this, she devised a system where presented images would first be pixelated to such an extent they could not be identified. Then, the number of pixels was increased over time until the participant could identify the object.

Recognition reaction times were compared for objects in three settings: a background devoid of information (without context), a familiar background in which the object would normally be found, and an unusual background. Barenholtz found that object identification was faster when the object was in a familiar context. Objects in an unusual context were identified more slowly, and those presented with no background were identified even more slowly. While the present study does not attempt to replicate reaction time to changes in pixilation, and therefore adopts a different design from Barenholtz's, it does base its hypothesis on her findings regarding object-context familiarity, seeking to determine the extent to which these translate to nonlocal perception.

\section{Objective}

The purpose of this exploratory project was to perform a comparative analysis of remote viewers' performances when tasked with describing an object placed in one of three different types of background. We also wanted to know whether remote viewers are more likely to describe the main target object compared to the setting of the object within the photographic image.

Hypothesis 1. Background/Setting within Photographs of Objects will make a difference to remote viewing success. Based on the theory that extrasensory perception mirrors physical sensory perception, it was hypothesized that experienced remote viewers would have most success viewing objects set within their natural or expected background. It was predicted that performance would decline for these experienced viewers when working with photographs of objects placed within a plain background devoid of information. This is based on the idea that most of the participants are trained in and/or utilize methodologies 
that encourage them to use imagery that has them visualizing they are moving around a location and positioning their awareness at different vantage points, while allowing sensory data to come in on a full body level through asking probing questions such as "what do I hear, feel, taste, smell, etc." We predicted that viewers would have the highest rate of incorrect information when the target object was placed in an unusual, confusing, or illogical background. This prediction was based on our theory that viewers tend to have greater instances of psi missing or distortion of information when they cannot reconcile confusing elements during a session, and on Barenholtz's findings that participants in her research had the most difficulty identifying objects placed in an unusual, confusing, or illogical background.

Hypothesis 2. Some participants will perform better than others. This is based on findings from past studies that certain remote viewers did consistently better in ongoing projects then others (Utts, 2018) and that "select subjects" did better than non-select (Storm \& Tressoldi, 2020). Further, a recent study found that had results been assessed individually, rather than collectively for the group of viewers, results (in terms of both hit/miss rate and amount of earnings from wagers made on predictions for sporting events) could have been significantly better (Katz et al., 2019a).

Hypothesis 3. Object Categorization. Given that all of our participants had substantially more experience with location-based targets than object-based targets, we hypothesized that more of their correct impressions would pertain to the background than to the main object. This was only relevant to two of the conditions, those with normal or abnormal backgrounds. Even though remote viewers were advised that all the targets contained objects as focal points, they were given permission to describe the entire photograph.

\section{METHODS}

\section{Participants}

Five men and seven women participated as remote viewers. Six had more than 10 years of remote viewing experience, four had 5 to 10 years, and two had 2 to 4 years. Three of the remote viewers had completed more than 1,000 sessions. Five had completed between 500 and 1,000 
sessions. Three had completed between 200 and 500 sessions. Two had completed between 20 and 99 sessions. Nine indicated they had been trained in Controlled Remote Viewing methodology (Smith, 2014) or a derivative. One had training in Extended Remote Viewing (Morehouse, 1998). The remaining two had unspecified training and approaches. Ten of twelve indicated that they did not have a lot of experience describing objects within photos, but they felt confident they could do so.

Traditionally in remote viewing research or operation projects, participants have been assigned viewer numbers (Smith, 2005). However, researchers felt numbers can have a dehumanizing effect. In order to give viewers a sense of empowerment and create a fun, positive environment, viewers were told to choose a God or Goddess name of their choice from any mythology or tradition. These names were used as participant identifiers to maintain confidentiality.

\section{Separation of Roles}

Researchers' roles were defined to ensure appropriate blindness at all phases of the project. Descriptions can be found in Table 1.

\section{Materials}

Materials included the photographic targets, score sheets for Phase I judging, and survey forms and photo sets for Phase II judging.

Targets. Targets were selected specifically for this project by the first author. 30 photographs were created and utilized, each containing a prominent single object clearly and immediately identifiable, even by cursory observation, as the center of interest. We referred to these center-of-interest objects as "main objects." Photographs were mostly collected from royalty-free Internet sites.

Object themes. The main purpose of the project was to compare remote viewing performance for target objects set within three different background conditions. Therefore, it was necessary to ensure that the photos to be compared would be similar in other ways. It would have been ideal to be able to use the exact same object across each different background condition, but this would have then meant we had the same ten objects occurring across three backgrounds. It was felt that this might produce cognitive noise narrowing the "free response" into more of a forced-choice task, if the viewers started to suspect that the 


\section{TABLE 1}

\section{Researchers' Roles and Responsibilities}

\section{Researcher Responsibilities}

Researcher \#1

(Katz)

Researcher \#2

(Bulgatz)

Researcher \#3

(Lane)

Randomizer

Key Holder
Initial project design, Initial recruitment of remote viewers and judges; designing the participation agreement and training materials; overseeing viewer and judge trainings; creation of the target pool; sending target descriptors to an individual we will refer to as "the Randomizer"; creation of the photo sets for Phase II judging, overseeing data entry into master spreadsheets after receiving all score sheets from Researcher \#2.

Oversaw the entire experimental phase of the project which included communicating with all potential subjects along with Phase I and Phase II judges, the randomizer, the score sheet creator, etc.; preparation and delivery of the score sheets; and serving as a liaison between these participants and Researcher \#1.

Served as a statistician for the overall project (in addition to and after having served as an independent judge for Phase I). He calculated all statistics and supplied the statistical portions of this paper and served as a scientific advisor.

This was the individual assigned to generate the random assigning of targets. He sent the list to Researcher \#2, communicated on a biweekly basis with Researcher \#2 by sending the photo feedback per trial according to schedule, etc.

The Director of the Rhine Research Center, John Kruth, accepted our request to hold the key in the event something should happen to the Randomizer. He was the only other person besides the Randomizer who ever had access to the target list of randomized numbers connected to their photo names, prior to the photo feedback being released. He had no other connection with the project, nor was he aware of the identity of any of the remote viewers.

same object was going to continue to reappear. Therefore, as the next best option, ten object themes were created, with the idea these would either include the same kind of object (but using a different photograph of that object type), or an object that was very similar in its construction, size, material makeup, purpose, emotional impact, etc., to the others in the theme.

For example, one theme was guns. A photo of a rifle in a hand in 
a nondescript room was chosen for the regular background; a machine gun underwater was chosen for the unusual background; and a photo of a colorful squirt gun against a white background was used for the third condition. For the musical instrument themes, a grand piano in a studio was used for the regular background; an upright piano located at a shoreline was used for the unusual background; and an accordion set against a white background was used for the third condition.

In constructing the target pool, attempts were made to balance gestalts of water, land, sky, movement, desert, indoors, outdoors, natural, manmade, human, animal, etc., so these did not appear too often. Many of the object selections were informed by findings in the parapsychological literature discussed above. For example, one theme included objects possessing thermodynamic qualities as defined by May (2011). In alignment with Bem's (2011) target pool we included a theme of guns (related to violence) and bras (related to sexual arousal). At the same time, we wanted to incorporate themes of objects that are often used as targets within informal associative remote viewing projects, such as food, animals, and transportation devices. Table 2 contains the object themes and types of objects used.

TABLE 2

Category Object Name and Type of Object Used

\begin{tabular}{|c|c|c|c|}
\hline Subject Type & White Background & Regular Background & Abnormal Background \\
\hline Guns & Squirt Gun & Rifle in Hand & $\begin{array}{l}\text { Machine Gun under } \\
\text { Water }\end{array}$ \\
\hline Bras & Spikey Bra & Pink Bra on Woman & Bra on Tree in Forest \\
\hline Instruments & Accordion & Piano in Studio & Piano on Beach \\
\hline Food & Chocolate Cake & Italian Food on Table & $\begin{array}{l}\text { Sushi Plate on Satellite } \\
\text { in Outer Space }\end{array}$ \\
\hline Cigarettes & Marijuana & $\begin{array}{l}\text { Cigarette in Human } \\
\text { Mouth }\end{array}$ & $\begin{array}{l}\text { Cigarette in Fish Mouth } \\
\text { on Land }\end{array}$ \\
\hline Bikes & Green Quad & $\begin{array}{l}\text { Tandem Bike with } \\
\text { Kids on Pavement }\end{array}$ & Bike in Water \\
\hline Buildings & Castle & House on Land & House in Sky \\
\hline $\begin{array}{l}\text { Explosions / } \\
\text { Fire }\end{array}$ & Exploding Balloons & $\begin{array}{l}\text { Nuclear Explosion in } \\
\text { Desert }\end{array}$ & Fire on Burning Man \\
\hline $\begin{array}{l}\text { Animals / } \\
\text { Birds }\end{array}$ & Parrot & Polar Bears on Ice & Penguins in Desert \\
\hline
\end{tabular}




\section{Backgrounds and Settings}

To test the extent to which differing backgrounds affected remote viewer performance to distinguish and parse prominent center-ofinterest objects ("main objects") against an array of backgrounds, three visual background conditions were selected.

White background: The target object is shown on a white background, devoid of information or context

Normal background: The target object is shown within a setting in which the object could typically be found (e.g., piano in a living room)

Unusual background: The target object is shown in an unusual setting, where the object would not typically be found (e.g., piano on a beach).

Inevitably, both normal and unusual backgrounds might contain additional secondary objects within them. We selected backgrounds such that these secondary objects would be much less attentionattracting than the main objects, so as to interfere as little as possible in the process.

\section{Blinding Protocols}

All remote viewers and those in contact with them were blind to the nature and content of the assigned targets for each trial. They were told the definition of objects was very broad and could include anything of an inorganic or organic nature, with no parameters in terms of size or subject matter. They were told this in advance so they could adjust their chosen methodology to the task. All participants and both Researchers \#1 and \#2 were blind to the order of the assigned targets. During data collection, Researcher \#1, who had constructed the target pool, intentionally did not communicate with the viewers. All communications were conducted between the viewers and Researcher \#2, who was blind to the nature of the target pool and to all targets.

\section{Randomization of Target Material}

After careful selection of the 30 targets, Researcher \#1 recruited a volunteer (referred to as the "Randomizer") with statistical experience 
who was willing to both randomize the targets and send the photo feedback to Researcher \#2, according to a predetermined schedule. The randomizer was provided the link to an online computer program designed for the purpose of randomizing remote viewing targets, created by P. J. Gaenir (2013).

The Randomizer was instructed to examine the list of target themes to ensure that the same object theme did not repeat in succession. In this respect the randomization was only partial. The randomizer was not given any directions as to how to perform the separation. The rationale behind this request was that remote viewers often experience a sense of confusion when they begin to get impressions which remind them of a target they recently worked with. They cannot discern if it is related to the present target, or if they are just still focused on, or remembering, a past target. This then becomes a psychological dilemma and often they may choose to not report the aspects that are too close to the target they just had. This is not an intuitive problem, but an analytic one. Although it would decrease the overall effectiveness of randomizing, it was felt there was far less to lose from this decision than there would be to have too many repeating elements in a consecutive order, which was likely to happen given that out of ten target types, each would be repeating three times.

Once the list of target numbers was generated by this Randomizer, the list of target numbers only (without the attached photos) was given to Researcher \#2.

Target numbers construction. Two-digit numbers were added on to the randomized numbers, according to the order they were assigned to viewers. So ol was added to the end of the first random number for the very first trial and 30 was added to the very last target number that was assigned to viewers. These created the target numbers the viewers would see and work with.

\section{Rationale for Participants Completing All Targets at the Same Time and in the Same Order}

After much consideration, it was decided that all participants would receive the same target numbers at the same time, thus also receiving the same feedback photo at the same time. It was felt this would create greater cohesiveness, while decreasing the possibility 
of displacement occurring to other feedback photos, which has been observed to be a problem in our other projects when different viewers are receiving different targets during the same time period and then submitting their results to the same researchers. "Displacement" or "displaced psi" is thought to occur when one attempts to utilize one's psi-based perceptions to describe a target but then inadvertently describes something else, such as another photo in the judging set or another target that will assigned for a later trial. (Carrington, 1940/1941; Milton, 1986a, 1986b). This decision was also related to feasibility issues and a desire to maintain double-blinding protocols as our highest priority. We had 30 defined targets total, which had taken quite a bit of time to assemble. The only way to ensure that Researcher \#2, who needed to have ongoing contact with the remove viewers, remained blind to all targets and even to the nature of the target pool, was to have her only see a single feedback photo per each trial, once all remote viewing transcripts and score sheets were submitted. Once she and the viewers saw a photographic target, it was not reused.

While decisions such as this have been criticized by some parapsychologists concerned with a stacking effect, according to Brier (1976), who first discussed the stacking effect in relation to forcedchoice task type experiments involving multiple trials in one setting (such as when a deck of 52 cards is being "guessed" at), there are sometimes valid reasons to assign all participants the same target types, in the same order, such as when doing otherwise would render a project unfeasible, and this should not disqualify a design as being flawed (Thouless \& Brier, 1970).

\section{Timing and Procedures}

Viewer location. All remote viewers completed their sessions by themselves, unmonitored, from the privacy of their homes.

Viewer instructions. Viewers were given the following instructions: "Describe the main object that appears within the parameters of the photo that you will be shown at feedback time that is connected to the same target number you will be provided." Based on an understanding of the participants' various mental imagery methods and approaches typically utilized in remote viewing, further instructions included: "You are free to 'go' to the object in its location, or you can describe the photo itself. Not 
all photos may have a place to 'go' to. If you 'go' to a location, try to remain within the parameters of the photo because only your impressions that can be judged via looking at the photo will be scorable."

\section{JUDGING PROTOCOLS AND ANALYSIS—PHASE I RATING AND PHASE II RANKING OF SESSIONS}

Two different modes of judging were performed at two different points in time. (The rationale for this is presented below in the Discussion section.) These were broken down into Phase I and Phase II, which used different approaches and were conducted at different time periods. Viewers were aware of Phase I but not of Phase II. Phase I included viewers as self-judges and then independent judges. Phase II only utilized independent judging.

\section{Phase 1 Judging}

The schedule for Phase I judging is displayed in Table 3.

Independent Phase I judges. Six independent, volunteer judges were recruited either by personal invitation, referrals, or from postings on remote viewing forums. Criteria for judging were prior experience with rating sessions, a background in remote viewing, or knowledge in research methodology. Each judge was assigned to two viewers throughout the duration of the trial and was responsible for rating all of their transcripts (a total of 60, 30 per viewer).

The rationale behind this was to increase the likelihood of judging consistency across all target types and categories. Since researchers have witnessed on multiple occasions in the past that some judges are more generous in their scoring while others are much more conservative, we were aware that this could play out in decreasing some individual viewers' overall performance ratings, while enhancing others. However, since it was the target categories and photos themselves we were ultimately testing as part of our main hypothesis, we went for this approach.

Phase I judging approach. This approach involved comparing every single descriptive item and sketch to the feedback image, designating each as "correct" or "incorrect." Judges used a rating sheet created by Alexis Poquiz. It is a simplified modification of his earlier system which 
TABLE 3

Schedule of Remote Viewing Sessions and Phase I Judging (Biweekly)

\begin{tabular}{|c|c|c|}
\hline Steps & Activity & Timing \\
\hline 1 & $\begin{array}{l}\text { Viewer was sent tasking email notifying } \\
\text { them of the target number and due date. }\end{array}$ & $\begin{array}{l}2 \text { to } 3 \text { days before due date, } \\
\text { which is either Tuesday or } \\
\text { Friday, by 9:00 a.m. PDT. }\end{array}$ \\
\hline 2 & $\begin{array}{l}\text { Viewer performs RV session, produces } \\
\text { a transcript on white paper. Fills in data } \\
\text { score sheet and emails transcript and } \\
\text { data sheet to Researcher \#2. }\end{array}$ & $\begin{array}{l}\text { No later than 9:00 a.m. PDT } \\
\text { on either Tuesday or Friday. }\end{array}$ \\
\hline 3 & $\begin{array}{l}\text { Researcher \#2 contacts the randomizer to } \\
\text { ask for the correct feedback photo to be } \\
\text { sent. Randomizer promptly responds. }\end{array}$ & $\begin{array}{l}\text { As soon as every viewer } \\
\text { (12) has turned in their } \\
\text { transcripts. }\end{array}$ \\
\hline 4 & $\begin{array}{l}\text { Researcher \#2 sends the feedback photo } \\
\text { instructing viewer to rate all perceptions } \\
\text { and sketches using the same data score } \\
\text { sheet for self-judging. }\end{array}$ & $\begin{array}{l}\text { As soon as every viewer } \\
\text { (12) has turned in their } \\
\text { transcripts. }\end{array}$ \\
\hline 5 & $\begin{array}{l}\text { Viewers self-judge and indicate on the } \\
\text { score sheet whether perceptions pertain } \\
\text { to object or background. Return the score } \\
\text { sheet. They then immediately turn in to } \\
\text { Researcher \#2. }\end{array}$ & $\begin{array}{l}\text { Viewers need to send in } \\
\text { prior to the start of the next } \\
\text { trial, so either by Monday or } \\
\text { Thursday. }\end{array}$ \\
\hline 6 & $\begin{array}{l}\text { Researcher \#2 hides/locks viewers self- } \\
\text { scores protected by a password and } \\
\text { forwards the score sheet to independent } \\
\text { judges. }\end{array}$ & $\begin{array}{l}\text { Sent to independent judges } \\
\text { once received from viewer. }\end{array}$ \\
\hline 7 & $\begin{array}{l}\text { Phase I independent judges make use } \\
\text { of the viewer's RV perceptions already } \\
\text { inputted into score sheet (so they don't } \\
\text { have to input themselves) but are blind to } \\
\text { the viewers' scores. They return the score } \\
\text { sheet to Researcher \#2. }\end{array}$ & $\begin{array}{l}\text { Returned to Researcher \#2 } \\
\text { prior to commencement of } \\
\text { next trial. }\end{array}$ \\
\hline
\end{tabular}

This completes the trial, and the process starts all over again. 
the present authors have referred to as the Poquiz Method of Scoring (Katz \& Bulgatz, 2013; Katz \& Knowles, 2021), which utilizes an Excel sheet that allows for various fields to be hidden and locked and for individual scores from columns to be automatically calculated. The sheet allowed for the scoring of individuals' words and sketches.

The Poquiz Scoring system (Poquiz, 2012a, 2012b) is based on a systematic interpretation of the traditional SRI 7-point scale (Targ et al., 1995). Poquiz's interpretation uses numerically defined levels based on percentage values of correct and incorrect matches and the percentage value of unknown matches. The core concept of this approach involves systematically listing out every single descriptor and sketch from the viewer's session into a spreadsheet. Each descriptor and sketch is then rated by a judge as "Yes," "No," or "Unknown." The unknown response means the judge cannot evaluate the perception, but that does not necessarily mean it is correct or incorrect. For example, if the photo is of an ocean and the viewer says they feel a breeze, or smell fish, or hear birds. If these are not in the photo some judges may feel they cannot evaluate such impressions, but the impressions are not necessarily wrong.

This Phase I judging was completed by the viewers (self-judging), followed by independent judging by others who evaluated the same items. Judging was completed for one trial before the next trial could begin. This approach provides no statistical means to determine whether scores differed from chance. However, it was possible to compare the proportion of "yes" ratings (hit rate) across the three background conditions.

\section{Phase II Judging}

Judges for Phase II analysis method (sum of ranks matching). Twelve judges with remote viewing experience were recruited. These included newer remote viewing students and those who had either served as viewers, judges, or managers in multiple projects over the course of many years. One Phase II judge was assigned to one viewer, so that the same judge rated all 30 transcripts for that viewer. Most completed these during a one-week period following the completion of all viewing trials. 
Phase II judging approach. Using standard matching procedures, the 12 judges compared the viewers' data sheets and sketches to a randomly selected set of four photos, rank ordering the matches from 1 through 4 (best match to worst match) using the sum of ranks method described by Solfvin et al. (1978).

Judging sets. The sets of four photos were created by Researcher \#1 (Katz). Three decoy photos were chosen from the same royaltyfree websites per the same rules as the original targets and combined with the target image into a set. While other experiments have often used the targets for one trial as decoys in another, researchers wanted to avoid the possibility of judges logically dismissing certain photo choices because they had seen them appear elsewhere. This was found to be a problem with early remote viewing experiments (Utts, 2018). For this reason, it was decided that photos in the judging sets would not repeat. Once all 30 sets of targets and decoys were created, these were randomized by an online survey program which automatically shuffled the sets into different positions for each judge.

Phase II judging sets followed the criteria for creating sets that have been outlined in the work of May et al. (1990) as far as the importance of photos to be orthogonally different, yet similar as far as their level of interest, emotionality, and in entropy. To ensure the photos within each set of four were different from each other, the following rules were established for creation of the sets: All photos in a set ideally should have different backgrounds in terms of all major and minor gestalts (water, land, structure, air). All objects should differ as much as possible in shape, color, size, texture, luminosity, patterns, number, concept, and function. To ensure that judges' choices were based on the quality of the remote viewing session rather than on clues that could skew results, each photo set contained different objects set within the same background type. For example, targets with white backgrounds were paired with three decoy photos of objects against white backgrounds.

\section{RESULTS}

\section{Summary of Remote Viewing Sessions}

A total of 360 remote viewing (RV) sessions were completed by the 12 viewers. Each viewer completed all 30 target images, ten in each of 
the three background conditions. Viewer transcripts provided a total of 8,460 descriptive items and 1,472 sketches for evaluation. The number of descriptive items and the number of sketches found within the remote viewing transcripts varied amongst the 12 viewers, with some providing many more than others. Table 4 summarizes these data.

\section{TABLE 4}

\section{Number of Descriptive Items and Sketches by Viewer over 30 Trials}

Values include Mean (SD) and minimum and maximum number.

Duration includes Mean (SD) and minimum and maximum time reported by the viewer.

\begin{tabular}{llll}
\hline Viewer ID & $\begin{array}{c}\text { Number of Descriptive } \\
\text { Items per Trial }\end{array}$ & $\begin{array}{c}\text { Number of Sketches } \\
\text { per Trial }\end{array}$ & $\begin{array}{c}\text { Duration of } \\
\text { Viewing (min) }\end{array}$ \\
\hline Akeru & $70.3(16.0) 24-107$ & $7.1(2.8) 3-14$ & $49.7(24.6) 10-130$ \\
Athena & $10.9(3.6) 4-21$ & $2.4(1.1) 0-4$ & $6.4(3.7) 2-18$ \\
Bucephalus & $28.6(6.0) 19-40$ & $1.9(0.3) 1-2$ & $11.4(1.5) 8-15$ \\
Chicchan & $23.4(5.2) 16-43$ & $4.2(1.1) 3-7$ & $7.0(2.2) 4-11$ \\
Hashem & $24.8(5.7) 15-35$ & $2.6(1.2) 1-5$ & $21.5(15.9) 10-86$ \\
Isis & $97.8(23.5) 53-139$ & $11.0(3) 6-16$. & $23.5(8.0) 12-40$ \\
Neptune & $19.9(4.2) 11-28$ & $2.9(1.1) 1-5$ & $12.8(3.6) 6-20$ \\
Nuadu & $18.8(5.7) 12-40$ & $3.6(1.1) 2-6$ & $26.0(5.7) 6-36$ \\
Parvati & $6.1(3.0) 1-12$ & $7.8(3.5) 2-15$ & $19.5(6.4) 8-30$ \\
Sulis & $38.1(5.5) 27-46$ & $1.0(0.0) 1-1$ & $23.7(5.7) 10-35$ \\
Tawa & $11.6(2.6) 7-17$ & $2.8(0.8) 1-5$ & $44.5(11.3) 28-70$ \\
Zeus & $10.0(2.4) 5-15$ & $1.9(0.9) 1-4$ & $9.4(3.4) 5-23$ \\
\hline
\end{tabular}




\section{Analysis of Sum of Ranks Scores (Phase II)}

Hypothesis \# 1-The primary hypothesis of the study was that the background in which an object is positioned would affect remote viewing success. This was confirmed. However, the prediction, based on visual perception research, that performance would be best in the normal background condition was not confirmed. Instead, for this form of judging involving a matching task activity, it was confirmed that trials containing objects shown on a white background were more successful than those with objects embedded within normal or abnormal conditions.

Repeated-measures analysis of variance of the target rank score for each transcript was conducted with a regression model including factors for background condition and target within condition (Proc Mixed, SAS ver. 9.4, SAS Systems, Cary, NC). Analysis revealed a significant difference among the three background conditions $(F(2,22)$ $=5.58, p=.01$ ). Pairwise comparisons of the three conditions revealed that the sum of ranks for the White background $(M=21.2, S E=0.97)$ was significantly lower than for the Normal background $(M=25.5, S E$ $=1.0)$. This difference was $4.3(S E=1.4)$, with $p=.005$. (Note: For those unfamiliar with this type of analysis, lower indicates a stronger effect.) Similar differences were found between the White background and the Abnormal background $(M=24.7, S E=1.0)$ with a difference of $3.5(S E=$ 1.4) and $p=.02$. However, sum of ranks scores did not differ between the Normal and Abnormal background conditions $(p=.5)$.

Hypothesis \# 2-The hypothesis that some participants would perform better than others also turned out to be correct. The overall success of remote viewing was assessed for each individual viewer, both within each of the three background conditions ( $N=10$ sessions) and across all three conditions ( $N=30$ sessions) using Monte Carlo simulations programmed in SAS ver. 9.4 (SAS Systems, Cary, NC).

Simulations based on 10,000 trials revealed that a sum of ranks $\leq 19$ exceeded the criterion for significance at $p \leq .05$ (one-tailed) for $\mathrm{N}$ $=10$ targets and four possible ranks. For the 36 combinations of viewer and background condition, only five of the calculated sums of ranks scores were found to be significantly smaller than chance. The lowest four sums of ranks were all in the White background condition, and 
the fifth was in the Abnormal background condition. When all 30 trials were evaluated, the simulation estimate for 30 trials and four ranks was sum of ranks $\leq 65$ for $p \leq .05$ (one-tailed), which only three of the 12 viewers achieved. These three participants achieved performance better than chance in both analyses, total and by condition.

\section{Analysis of Phase I Judging-Item Hit Rate/Transcript Analysis}

The secondary objective of the project was to explore an alternative procedure for evaluation of the RV transcripts, which assigned each descriptive item and sketch in the transcript a designation of "hit" or "miss" based on its relevance to the target object. Scores for each transcript were summarized for each target as the proportion of correct scores ("hit rate"), with separate hit rates calculated for descriptive items and for sketches. These two hit rates were averaged to yield a combined score that weighted them equally, despite the much higher number of descriptive items. Separate scores were created for the viewer (selfjudging) and independent judge ratings that scored each transcript.

Separate repeated-measures analyses of variance were conducted for the viewer self-ratings and the independent judge ratings (SAS 9.4, SAS Institute, Cary, NC). Results for these analyses were mixed. For viewer self-rating scores, the effect of background condition was significant for the combined hit rates $(F(2,22)=4.53, p=.02)$. Pairwise comparisons of the conditions for viewer self-ratings indicated that hit rates for the Normal and Abnormal backgrounds were significantly higher than for the White background (see Table 3). This effect was opposite to that found in the analysis of sum of ranks scores.

However, for the independent judges' scores, the effect of condition was not significant $(F(2,22)=1.76, p=.19)$. Results are summarized in Table 5 .

Hypothesis \#3-object categorization. Our hypothesis that more of the viewers' correct impressions would pertain to the background than to the main object (based on the premise they are more experienced describing locations than objects) is rejected. Results indicated that viewers more often described the object than the background. We had hypothesized the opposite result would be true, based on our viewers' responses during a pre-experimental survey that they were more experienced with describing locations. 


\section{TABLE 5}

Effects of Background Condition on Viewer and Judge Ratings (Hit Rate) of Descriptive Items and Sketches for $\mathrm{N}=30$ Targets in Three Background Conditions Combined ratings equally weight items and sketch ratings for each viewer and trial.

\begin{tabular}{lllcl}
\hline \hline & $\begin{array}{c}\text { Condition } \\
\text { Effect } \\
d f=(2,22)\end{array}$ & $\begin{array}{c}\text { Normal } \\
\text { Background } \\
\text { M (SEM) }\end{array}$ & $\begin{array}{c}\text { Abnormal } \\
\text { Background } \\
\text { M (SEM) }\end{array}$ & $\begin{array}{c}\text { White } \\
\text { Background } \\
\text { M (SEM) }\end{array}$ \\
\hline $\begin{array}{c}\text { Viewers } \\
\begin{array}{c}\text { Combined } \\
\text { Hit Rates }\end{array}\end{array}$ & $\begin{array}{l}F(2,22)=4.53, \\
\mathrm{p}=.02\end{array}$ & $0.61(0.05)$ & $0.62(0.05)$ & $0.53(0.05)$ \\
$\begin{array}{c}\text { Judges } \\
\text { Combined } \\
\text { Hit Rates }\end{array}$ & $\begin{array}{l}F(2,22)=1.76, \\
\mathrm{p}=.19\end{array}$ & $0.43(0.05)$ & $0.46(0.05)$ & $0.41(0.05)$ \\
\hline
\end{tabular}

Instructions for item scoring defined a hit as the relevant presence of the descriptor or sketch to any part of the target image. Using the data available for independent judges, each hit was assigned to one of four categories related to: the main object of the target; the background of the object; a non-essential or peripheral object in the image; or more than one of these.

Because trials yielded widely varying numbers of item and sketch hits, the number of hits assigned to each category was converted to a proportion of the total for that trial.

Table 6 summarizes the distribution of these data. Category proportions for each trial were averaged for each viewer, using all available trials. The viewer averages were then combined into an overall average for all viewers. A total of 207 trials were evaluated in this manner. The overall averages indicated that the majority of items were determined to be associated with the main object, with an average across viewers of 0.64 . Much smaller proportions of items were associated with the image background (0.09), non-essential objects in the image (0.14), and more than one category (0.17). The results for individual viewers in Table 6 suggest large variations among viewers in the proportion of items specific to the target object. 
TABLE 6

Average Proportions of Transcript Items (Descriptors and Sketches) Relevant to Four Categories within the Target Image by Viewer

$\mathrm{N}=$ number of targets included for each viewer.

\begin{tabular}{|c|c|c|c|c|c|}
\hline Viewer & $N$ & Main Object & Background & $\begin{array}{c}\text { Non-Essential } \\
\text { Object }\end{array}$ & $\begin{array}{c}\text { Multiple } \\
\text { Categories }\end{array}$ \\
\hline
\end{tabular}

\begin{tabular}{|l|c|c|c|c|c|} 
Akeru & 17 & 0.70 & 0.06 & 0.20 & 0.03 \\
Athena & 13 & 0.54 & 0.20 & 0.12 & 0.21 \\
Chicchan & 16 & 0.66 & 0.22 & 0.18 & 0.30 \\
Hashem & 18 & 0.45 & 0.03 & 0.24 & 0.18 \\
Isis & 18 & 0.72 & 0.06 & 0.14 & 0.40 \\
Neptune & 18 & 0.53 & 0.13 & 0.21 & 0.03 \\
Nuada & 18 & 0.66 & 0.03 & 0.13 & 0.09 \\
Parvati & 17 & 0.98 & 0.07 & 0.05 & 0.09 \\
Sulis & 18 & 0.86 & 0.05 & 0.15 & 0.13 \\
Tawa & 18 & 0.48 & 0.04 & 0.10 & 0.02 \\
Zeus & 18 & 0.67 & 0.08 & 0.06 & 0.23
\end{tabular}

Comparisons of hit rates for viewers and independent judges. It was found that viewers acting as self-judges scored more of their own words and sketches higher than the independent judges did when rating the same information.

The difference between viewer and independent judge hit rate for each target was determined and summarized across targets (Table 7). Evaluation of the differences by $t$ test revealed statistical significance for 5 of 12 viewers, all with higher hit rates for viewers. Comparing the average difference for all targets across the group of 12 viewers by 
paired $t$ test revealed that viewers' hit rates $(M=0.58)$ were significantly higher than those for independent judges $(M=0.43)$ with $t(11)=2.99$, $p=.01$.

The retest reliability of hit rates for viewers and judges, which is a measure of the consistency of scores between these two categories of raters, was evaluated by correlation/regression using the mean values for each viewer $(\mathrm{N}=30$ trials). The correlation between mean values of hit rates for viewers and judges was not significant $(r=0.33, p=.30)$. The linear regression of viewer mean scores on judges mean scores yielded $F(1,10)=1.18, p=.30$, with $R^{2}=0.10$. Results show that mean hit rates for viewers and judges were unrelated and seemingly independent of one another.

TABLE 7

Comparisons of Target Hit Rates for Viewers and Independent Judges

\begin{tabular}{|c|c|c|c|}
\hline \multirow[t]{2}{*}{ Viewer } & \multicolumn{2}{|c|}{ Mean Hit Rate ( $N=30$ trials $)$} & \multirow[t]{2}{*}{ Test of Mean Difference $t(29),(p)$} \\
\hline & $\begin{array}{l}\text { Viewer Self- } \\
\text { Rating }\end{array}$ & $\begin{array}{l}\text { Independent } \\
\text { Judge }\end{array}$ & \\
\hline Akeru & 0.73 & 0.25 & $11.5(<.00000)$ \\
\hline Athena & 0.64 & 0.57 & $1.1(.27)$ \\
\hline Bucephalus & 0.45 & 0.42 & $0.6(.57)$ \\
\hline Chicchan & 0.36 & 0.40 & $-1.3(.22)$ \\
\hline Hashem & 0.73 & 0.42 & $8.5(<.00000)$ \\
\hline Isis & 0.56 & 0.15 & $9.9(<.00000)$ \\
\hline Neptune & 0.46 & 0.47 & $-0.2(.88)$ \\
\hline Nuadu & 0.57 & 0.47 & $1.7(.11)$ \\
\hline Parvati & 0.59 & 0.61 & $-0.4(.70)$ \\
\hline Sulis & 0.78 & 0.71 & $1.3(.22)$ \\
\hline Tawa & 0.42 & 0.26 & $3.8(<.001)$ \\
\hline Zeus & 0.75 & 0.48 & $5.3(<.00001)$ \\
\hline
\end{tabular}


Relationship of Phase 1 hit rate and Phase 2 rank. The relationship between the viewer and independent judge hit rate score and matching rank were evaluated using repeated-measures analysis with Proc Mixed (SAS 9.4, SAS Institute, Cary, NC) with factors for trial and viewer. Statistically significant effects of rank were observed for viewer and independent judge hit rates. Results are summarized in Table 8. Estimated hit rates from the models indicate that hit rate was highest for targets given the best (lowest) rank and decreased monotonically as the rank increased. These results provide supportive evidence of a relationship between the two methods of judging for the trial transcript.

TABLE 8

Relationship between Phase 1 Hit Rate and Phase 2 Rank for Target Match

\begin{tabular}{|c|c|c|c|c|c|}
\hline & \multirow[t]{2}{*}{$F(3,33)(p)$} & \multicolumn{4}{|c|}{ Estimated Hit Rate by Match Rank (M (sem)) } \\
\hline & & Rank 1 & Rank 2 & Rank 3 & Rank 4 \\
\hline $\begin{array}{l}\text { Viewer } \\
\text { Self-Rating }\end{array}$ & $6.20(.002)$ & $0.64(0.04)$ & $0.62(0.05)$ & $0.55(.05)$ & $0.50(0.05)$ \\
\hline $\begin{array}{l}\text { Independent } \\
\text { Judge }\end{array}$ & $3.70(.02)$ & $0.48(0.05)$ & $0.46(0.05)$ & $0.42(0.05)$ & $0.37(0.05)$ \\
\hline
\end{tabular}

\section{DISCUSSION}

Rationale for Including Two Different Types of Analysis \& Self-Judging for Phase

When first designing the study, researchers wanted to avoid a method of analysis that would require the use of photo sets involving decoys, due to the extent of displacement they felt they had personally witnessed in participating in dozens of other projects utilizing matching tasks methods.

Displacement is thought to occur when a psi percipient who is attempting to obtain information about a target or subject matter instead accesses information that is spatially or temporally removed from the designated target. This effect has been observed by numerous 
researchers, starting as early as 1884 when Richet found a decline in results not due to lack of the existence of psi but because of "consistent confusions of one target for another" (Alvarado, 2008, p. 543). He was followed by others such as Bruck (1925) in relation to hypnotized subjects and Sinclair (1930) in relation to subjects performing telepathic and clairvoyant tasks who sometimes described a photo intended for a future date.

The actual term "displacement" or "displacement effect" was coined by Carrington in 1940, who ultimately concluded, "On the whole I think there is very little to be said about forced matching for this purpose, or any other.... it is liable to be completely wrecked from the phenomenon of displacement" (p. 74). J. B. Rhine in 1950 wrote that "Pratt and Foster of the Duke Laboratory have recently found that the subject's displacement and consistent missing combined may produce a highly complex pattern of significant effects" (Pratt et al., 1940). In 1953, Rhine's wife, Louisa, turned away from forced guessing experiments, opting for a qualitative focus, explaining "Over the years, subjects in tests have seldom made perfect scores, and such frustrating effects such as displacement and psi missing have frequently been encountered" (Rhine, 1962a, 1962b). Tart (1980) explored this topic further in a paper titled, "Are we interested in making Psi function strongly and reliably?" also arguing against the use of matching tasks. According to Milton (1986a, 1986b), Tart's paper reinvigorated interest in the topic of displacement and it also led to her writing an entire dissertation on the subject. The present authors also have observed such effects (Katz et al., 2017) and as a result were determined to find a way to mitigate it in the present study.

The present researchers sought to eliminate all factors that might lead to this phenomenon. However, during their proposal review, advisors felt that a standard matching task would be important in terms of statistical analysis. Therefore, we came up with a compromise to ensure that the viewer's attention would not be likely to move to the wrong photos in a set. We created two phases of judging, and only informed the remote viewers of the first phase. We instructed the viewers that immediately upon receiving their feedback, they would self-judge through rating every impression as correct or incorrect to form a hit-rate score. 
This would require the viewers to spend a lot of time studying their feedback photo. While there is some recent evidence that suggests feedback may not be necessary (Müller et al., 2019), we theorized intensive engagement with the correct future feedback photo might strengthen target contact as is commonly asserted by those involved in applied projects (Katz \& Knowles, 2021).

Viewers were advised that independent raters would also rate their responses and sketches in a similar manner, within 48 hours of when they completed their judging. In this way, all participants' attention was carefully directed and kept away from the Phase II Sum of Ranks matching tasks that did use sets of four photos, with only one being the correct target. To create even greater distance in time and space, Phase II judging was performed by a whole different team of judges than Phase I, and only occurred after the entire experimental phase and Phase I judging were completed. To this day, most of our viewers still are not aware that there was a second phase of judging that involved matching tasks and decoy photos.

\section{Did We Manage To Avoid Displacement?}

We did a sampling of the Phase II judging responses for those trials that resulted in misses, and polled the judges, looking to see if there were very close matches to the wrong photo in the sets. We did not find any examples of this. Since we can't know if there would have been displacement if only Phase II judging had been utilized, we can't say for sure that our approach of using two judging methods did in fact reduce displacement, but this approach does seem promising. Future projects could test this by having one group of viewers who are made aware of matching-task independent judging and another group blind to the procedure to see if there were differences.

Still, we (and some of our viewers) may have seen examples of temporal displacement to targets that appeared later (Crandall \& Hite, 1983). One viewer felt this was happening quite a bit, and reviewing her session work we agreed with a few of her examples. One viewer in an early trial drew a picture of an accordion that was almost an identical match to the photo of an accordion against a white background. The odd thing was this picture did not appear in the sequence until several weeks later (Appendix Exhibit F). We cannot say of course if this was a 
displacement effect or simple coincidence but the sketch for trial 6 and feedback photo for trial 16 are strikingly close.

\section{Differences Found in Judging Methods}

We are somewhat perplexed at the opposite findings on the effects of background condition obtained by different judging methods (Phase I hit rates vs. Phase II Sum of Rank Matching Tasks). One possible explanation for this is that when it comes to matching tasks, there are possibly different perceptual and cognitive processes involved for judges. Both forms of analysis involve comparing impressions and sketches to a photo, but a rank ordering matching task involves repeating these comparisons multiple times per each photo in the set, and then having to make a number of decisions and choices. Given the complexities involved, it may be that it is simply easier for judges to make sense of data when there is no information in the background, hence the greater success with the white background conditions.

Regardless of the reason for the condition differences, these results suggest that if only a hit-rate type of approach is being used, it may be advisable to use photos of objects within their normal or abnormal backgrounds and to avoid the use of photos in white backgrounds. Conversely, if a project is going to use a matching sum of ranks form of analysis, it may then be best to use targets of objects against white backgrounds. A word of caution though: There are many designs within parapsychology experiments that use matching tasks but do not use rank ordering-meaning there will be only one score given to the best match, and no credit given to second-best match. We don't know if we would have obtained the same results using an alternative approach.

\section{Differences Found between Self-Judging and Independent Judging}

For our Phase I judging protocols (hit rates), 9 out of 12 viewers acting as self-judges (following submission of their transcripts to researcher 2) more frequently scored their own words and sketches correctly then did the independent judges when rating the same information. Also, three of the four viewers who generated the highest number of sketches and words produced the greatest differences between rater self-scores and independent judges scores. We cannot say if this is due to a desire to rate themselves more highly, or because 
they had a better ability to know what they meant both from their words and from their own sketches. Given that viewers were required to input their words into spreadsheets and submit these along with their transcripts prior to receiving feedback and self-scoring, we are confident it was not the case that they simply added in data after they received feedback.

\section{Rater Reliability}

For Phase I judging, the choice was made to have the same judge rate all 30 transcripts for the same two viewers so there would be consistency of judging background conditions, since this was the main focus of our exploration. We felt the judges did maintain intra-judging consistency, with little variability observed across trials. Although we provided training sessions for our judges that included verbal and written instructions, we did notice variability in judging styles between judges. We performed an informal test of inter-rater reliability for several of the viewers' sessions that had the greatest disparity between viewer's self-scores and independent rater scores, finding that our own scoring would have fallen somewhere in the middle of these scores. Therefore, we feel it was not appropriate to compare hit rates among viewers or to make definitive statements about their performance for this method of judging. One way around this issue would have been to assign the same judge to rate the transcripts of all 12 viewers, for the same trial. Then to maintain consistency across background conditions, the same judge would have to repeat the judging for all 12 viewers when the same object theme appeared two more times. Given the time-consuming nature of scoring all words and sketches, this may have been too formidable a task for some judges, although perhaps not so much so that we'd rule it out if we repeated the study.

Viewers described the main object. We originally hypothesized that given that the remote viewers are more experienced with describing locations, we expected they might be likely to describe locations rather than the main object. We hoped this was not the case, but just expected it to be, especially since over the years we have heard viewers express a dislike for photographs of objects rather than locations. They were instructed that the target pool consisted of objects within a variety of locations, and that their goal was to describe the main object, but that 
all correct information pertaining to every element (whether object or background) would be scored as correct. They proved us wrong, as results indicated they did most often describe the main object. However, since they knew that the photos did all involve a main object, we cannot say whether their own perception naturally and involuntarily went to these objects, or whether they directed themselves through voluntary attention (Ribot, 1903) to focus on the objects themselves.

This brings us to one of the main aims of our study: Did results correlate with Barenholtz' "normal" visual perception study findings? Again, for item/sketch hit rates it did, for matching sum of ranks tasks it did not. One may want to keep in mind that her design involved single participants attempting to recognize objects set within different backgrounds as pixels were increased as their response times were measured. There was no judging involved and hence no perceptual aspects of judges to potentially impact the process.

\section{In Conclusion}

Throughout our literature review two theoretical assumptions seemed to motivate investigations into target characteristics. The first was that a universalized set of characteristics for target material could, or should, be found, to enhance the non-local perception of all participants. The second was an acknowledgement that target characteristics may be more individualized, having a different effect on different viewers based on factors such as personal preferences, interests, experiences, and personality traits.

Given that only a few of our experienced remote viewers who have reputations for doing well at location-based targets achieved significant results for these object-oriented targets, future researchers should not expect that a remote viewer who performed well in one project with a completely different set of targets and protocols, will necessarily perform the same way with other materials and protocols.

Object categories and future research. Finally, we'd just like to say a bit more about the object categories. As noted above, choices were made to include some objects as targets based on past parapsychological literature demonstrating that arousing images (such as those of a sexual or violent nature or having more numinosity or entropy) may produce a stronger effect. These include bras, cigarettes, 
and guns and exploding items. Since comparing types of objects was not part of our original plan, we did not do a formal analysis of which targets produced the best results overall. However, we had a couple of volunteers go through all transcripts and visually choose which images stood out as the most striking matches to the target photos. In our Appendix we present a few of these. Exhibits $A$ and $C$ show examples of sketches related to our smoking targets. Exhibit B shows sketches for the nuclear explosion target, and Exhibit D shows examples of sketches produced in relation to the guns. One viewer referred to the older rifle as a "collector gun." Exhibit E shows one of our food targets, a cake, which some viewers we know anecdotally have shared they tend to miss. That viewer correctly identified it as a "cake," although misidentified it as a wedding cake instead of a birthday cake. What these examples suggest is that the target pool that was specifically created for this project may hold merit for other projects, whether simply to use as targets or to be compared to each other to see which produce higher hit rates based on the object content alone. These can be made available to other serious researchers upon request.

\section{ACKNOWLEDGMENTS}

This project was a recipient of the IRVA/IRIS Warcollier Proposal Award. Special thanks to Dr. Paul H. Smith for his editorial assistance.

\section{REFERENCES}

Alvarado, C. (2008). Note on Charles Richet's “La Suggestion Mentale et le Calcul des Probabilits" (1884). Journal of Scientific Exploration, 22(4), 543-548.

Barenholtz, E. (2013). Quantifying the role of context in visual object recognition. Visual Cognition, 22(1), 30-56. https://doi.org/10.1080/13506285.2013.865694

Bem, D. J. (2011). Feeling the future: Experimental evidence for anomalous retroactive influences on cognition and affect. Journal of Personality and Social Psychology, 100(3), 407-425. 10.1037/aoo21524

Brier, R. M. (1976). Correspondence ESP experiment with high I.Q. subjects. Journal of Parapsychology, 31(2), 143-148.

Bruck, C. (1925). Experimentelle telepathie. Proceedings, 35, 466-469. BR/PSI-X/ telepath/France.

Carrington, W. (1940/1941). Experiments on the paranormal cognition of drawings. Proceedings of the Society of Psychical Research, 46, 34-151. 
Crandall, J. E., \& Hite, D. D. (1983). Psi-missing and displacement: Evidence for improperly focused psi? Journal of the American Society for Psychical Research, $77(3), 209-288$.

Delanoy, D. L. (1989). Characteristics of successful free-response targets: Experimental findings and observations. In L. A. Henkel \& R. E. Berger (Eds.), Research in Parapsychology 1988 (pp. 92-95). Scarecrow.

Gaenir, P. J. (2013). Target reference number generator. http://www.learnrv.com/ generator/

Honorton, C. (1985). Meta-analysis of psi ganzfeld research: A response to Hyman. Journal of Parapsychology, 49(1), 51-91.

Honorton, C., \& Schechter, E. I. (1987). Ganzfeld target retrieval with an automated testing system: A model for initial ganzfeld success. In D. H. Weiner \& R. D. Nelson (Eds.), Research in Parapsychology 1986 (pp. 36-39). Scarecrow.

Honorton, C., Berger, R. E., Varvoglis, M. P., Quant, M., Derr, P., Schechter, E. I., \& Ferrari, D. C. (1990). Psi communication in the ganzfeld: Experiments with an automated testing system and a comparison with a meta-analysis of earlier studies. Journal of Parapsychology, 54(2), 99-139.

Katz, D. L., \& Bulgatz, M. (2013, Spring/Summer). Remote viewing the outcome of the 2012 presidential election. Aperture Magazine, 46-56.

Katz, D. L., \& Knowles, J. (2021). Associative remote viewing: The art a science of predicting outcomes for sports, politics, finances and the lottery. Living Dreams.

Katz, D. L., Bulgatz, M., \& McLaughlin-Walter, N. (2017). Predicting the 2016 U.S. Presidential Election using a double blind associative remote viewing protocol. Eight Martini's, 15, 4-15.

Katz, D. L., Grgic, I., \& Fendley, T. W. (2018a). An ethnographical assessment of Project Firefly: A yearlong endeavor to create wealth by predicting FOREX currency moves with associative remote viewing. Journal of Scientific Exploration 32(1), 21-54.

Katz, D. L., Grigc, I., \& Tressoldi, P. (2018b). The ARV rejudging project. Conference Proceedings of the Journal of Parapsychology, 82(2), 118-119.

Katz, D. L., Smith, N., Bulgatz, M., Graff, M., Graff, D., \& Lane, J. (2019a). The Associative Remote Dreaming Experiment: A novel approach to predicting future outcomes of sporting events. Journal of the Society for Psychical Research, 83(2), 65-84.

Katz, D. L., Bulgatz, M., \& Targ, R. (2019b). The other half of Hella's brain-An intimate conversation with Russell Targ. Eight Martini's: The State of the Art of Remote Viewing, 19, 4-20.

Krippner, S., \& Zeichner, S. (1974). Descriptive analysis of art prints telepathically transmitted during sleep. In W. G. Roll, R. L. Morris, \& J. D. Morris (Eds.), Research in Parapsychology 1973 (pp. 27-28). Scarecrow Press.

Krippner, S., Saunders, D., Morgan, A., \& Quan, A. (2018). Remote viewing of concealed target pictures under light and dark conditions. Explore: The Journal of Science and Healing, 15(1), 27-37. doi: 10.1016/j.explore.2018.07.001 
May, E. C. (2011). Possible thermodynamic limits to anomalous cognition: Entropy gradients. Journal of the Society for Psychic Research, 75, 65-75.

May, E. C., \& Lantz, N.D. (1991). Target and sender dependencies in anomalous cognition. Presented to the Scientific Oversight Committee. SAIC.

May, E., Utts, J. M., Humphrey, B. S., Luke, W. W., Frivold, T. J., \& Trask, V. (1990). Advances in remote viewing analysis. Journal of Parapsychology, 54(3), 193-228.

May, E. C., Spottiswoode, D. J. P., \& James, C. L. (1994a). Shannon entropy: A possible intrinsic target property. Journal of Parapsychology, 58, 384-401.

May, E. C., Spottiswoode, S. J. P, \& James, C. L. (1994b). Managing the target-pool bandwidth: Possible noise reduction for anomalous cognition experiments. Journal of Parapsychology, 58(3), 303-313.

Milton, J. (1986a). A critical review on the displacement effect. Proceedings of the Society for Psychical Research 1928-1952 [notes with mentions of displacement] https://www.cia.gov/library/readingroom/docs/CIA-RDP9600792Ro00701040005-1.pdf

Milton, J. (1986b). Thesis on displacement. Proceedings of the Society for Psychical Research 1928-1952 [notes with mentions of displacement]. Journal of Society for Psychical Research 1884-1952. https://www.cia.gov/library/readingroom/ docs/CIA-RDP96- 00792Ro00701040005-1.pdf

Mitchell, J. L. (1981). Verbal and performance skills in relation to ESP. [Ph.D. Disssertation, City University of New York]. https://www.proquest.com/ openview/ab37767d3978277465acc8f2eoa5a4be/1?pq-origsite=gscholar\&cbl= $18750 \&$ diss $=y$

Mitchell, J. L. (1988). Out-of-body experiences: A handbook. Aquarian Press.

Morehouse, D. (1998). Psychic warrior: The true story of America's foremost psychic spy and the cover-up of the CIA'S top secret Stargate Program. St. Martin's.

Morris, R. L. (1977). The Airport Project: A survey of the techniques for psychic development advocated by popular books. In J. D. Morris, W. G. Roll., \& R. L. Morris (Eds.), Research in parapsychology (pp. 54-56). Scarecrow Press.

Müller, M., Müller, L., \& Wittmann, M. (2019). Predicting the stock market. An associative remote viewing study. Zeitschrift für Anomalistik, 19, 326-346.

Nash, C. S., \& Nash, C. B. (1961). An ESP experiment with targets that differ in degree of similarity. Journal of the American Society for Psychical Research, $55,73-76$.

Poquiz, A. (2012a). Dung Beetle Scoring System (aka Poquiz Rating Method). http:// www.appliedprecog.com/\#!Dung-Beetle-Scoring-System-AKA-PoquizRating-Method/cut6/252C8A56-B4A4-4EBo-8754-4642 $\mathrm{D}_{71} \mathrm{C}_{125} \mathrm{E}$

Poquiz, A. (2012b). Alexis Poquiz Capstone. https://www.youtube.com/watch?v=STGIKHbBUio Pratt, J. G., Smith, B. M., Rhine, J. B., Stuart, C. E., \& Greenwood, J. A. (1940). Extra-sensory perception after sixty years: A critical appraisal of the research in extra-sensory perception. Henry Holt.

Rhine, L. (1962a). Psychological processes in ESP experiences: Part I. Waking experiences. Journal of Parapsychology, 26, 88-111. 
Rhine, L. (1962b). Psychological processes in ESP experiences. Part II. Dreams. Journal of Parapsychology, 26, 172-199.

Ribot, T. (1903). The psychology of attention. Forgotten Books. [Authorized trans.]]

Rosenblatt, M. (2000). Applications: AVM Precognition Project: Summary of results for protocol-1. Connections Through Time, 7(April/June). http://p-i-a. com/Magazine/Issue7/Applications_7.htm

Rosenblatt, R., Knowles, J., \& Poquiz, A. (2015). Applied Precognition Project (APP) and a summary of APP-2014. Connections Through Time, 38(April 2015-March 2016). http://p-i-a.com/Magazine/Issue38/Connections_38.html

Schmeidler, G. (1977). Letter from Schmeidler to Ingo on relationship between psi and perception. University of West Georgia Special Collections. https:// uwg.galileo.usg.edu/repositories/2/resources/274

Schwartz, S. A. (1977). Two application-oriented experiments employing a submarine involving a novel remote viewing protocol, one testing the ELF hypothesis. Journal of the Society for Psychical Research, 83(2), 84. doi: $10.13140 / R G .2 \cdot 2.22217 .95846$

Schwartz, S. A. (2019). The location and reconstruction of a Byzantine structure in Marea, Egypt, including a comparison of electronic remote sensing and remote viewing. Journal of Scientific Exploration, 33(3), 451-480.

Sinclair, U. (1930). Mental radio. Hampton Roads.

Smith, P. H. (2005). Reading the enemy's mind. Forge Books.

Smith, P. (2014). CRV-Controlled remote viewing: Manuals, collected papers \& information to help you learn controlled remote viewing. Amazon Digital Services.

Solfvin, G. F., Kelly, E. F., \& Burdick, D. S. (1978). Some new methods of analysis for preferential-ranking data. Journal of the American Society for Psychical Research, 72(2), 93-109.

Storm L., Tressoldi P. E., \& Di Risio, L. (2010). Meta-analysis of freeresponse studies, 1992-2008: Assessing the noise reduction model in parapsychology. doi:10.1037/ao019457

Swann, I., \& Puthoff, H. (1987). Natural ESP: The ESP core and its raw characteristics. Bantam Books.

Targ, R., \& Puthoff, H. E. (1974). Information transfer under conditions of sensory shielding. Nature 252, 602-607.

Targ, R., \& Puthoff, H. E. (1977). Mind reach. Delacorte Press.

Targ, R., \& Puthoff, H. (2005). Mind-reach: Scientists look at psychic abilities ( $2^{\text {nd }}$ ed.). Hampton Roads.

Targ, R., Kantra, J., Brown, D., \& Wiegand, W. (1995). Viewing the future: A pilot study with an error-detecting protocol. Journal of Scientific Exploration, 9(3) 367-380.

Tart, C. T. (1980). Are we interested in making ESP function strongly and reliably? A reply to J. E. Kennedy. Journal of the American Society for Psychical Research, $74(2), 210-222$. 
Thorpe, S. (2013). J. B. Rhine banquet address. Conference proceedings 56 th Annual Convention of the Parapsychological Association, Viterbo, Italy.

Thouless, R. H., \& Brier, R. M. (1970). The stacking effect \& methods of correcting for it. The Journal of Parapsychology 34(2), 124-128.

Tressoldi, P. E., \& Storm, L. (2020). Stage 1 registered report: Anomalous perception in a Ganzfeld condition: A meta-analysis of more than 40 years investigation. F1000 Research, 9, 826). https://www.ncbi.nlm.nih.gov/pmc/ articles/PMC7883320.3/

Utts, J. (2018). An assessment of the evidence for psychic functioning. Journal of Parapsychology, 82, 118-146. https://67f44e9a-5796-416c-b911-abd91a17obd3. filesusr.com/ugd/82c4a8_1ooed65cae6649949ca3ddd6eoefo527.pdf

Warcollier, R. (1938). Experiments in telepathy. Harper.

Warcollier, R. (1948). Mind to mind. Hampton Roads Publishing.

Watt, C. A. (1989). Characteristics of successful free-response targets: Theoretical considerations. In L. A. Henkel \& R. E. Berger (Eds.), Research in parapsychology (pp. 95-99). Scarecrow.

Williams, L. V., \& Siegel, D. S. (2014). The Oxford handbook of the economics of gambling. OUP.

\section{APPENDIX}

\section{EXHIBIT A}
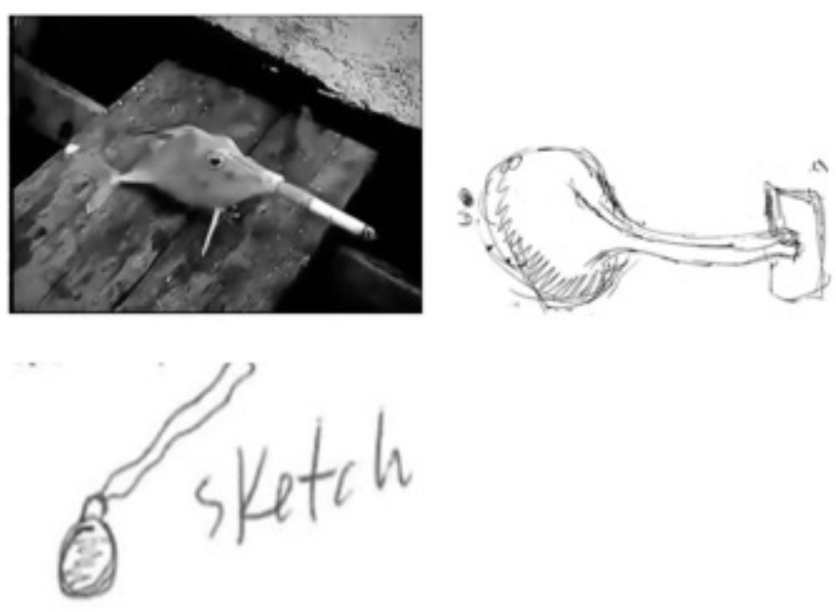

Target 1F13U006

Colors: Blues, rust, yellowish-gold, wheat, doody, white, striped, bright."

"Shiny, sticky, wet, moist, lifeform"; "reminds me of a whale, spongey, like a gold medal."

"Smoke smell, billowing". 


\section{EXHIBIT B}
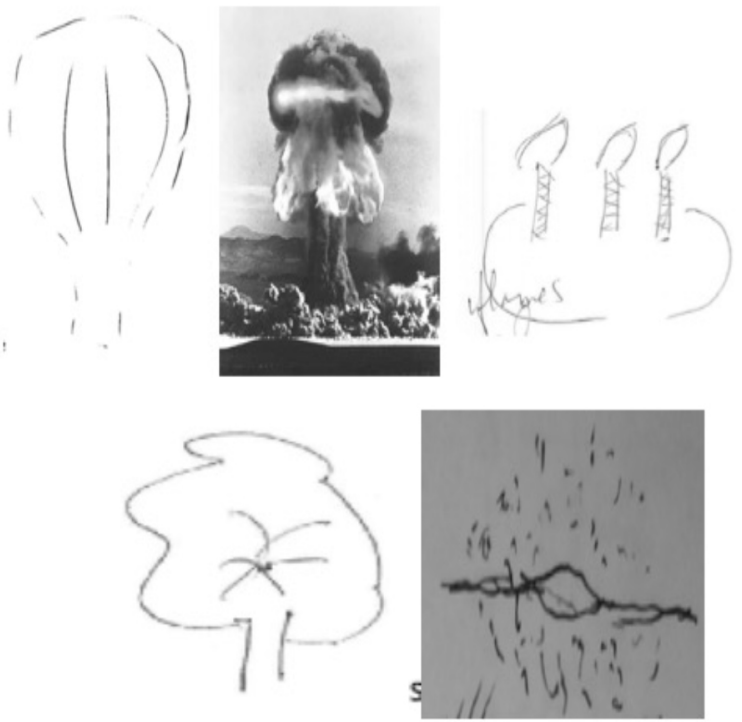

"Flames, hot, fiery, colorful, vibrant, ballistic, military, woozy, vertigo, mortar, funnels"

\section{EXHIBIT C}
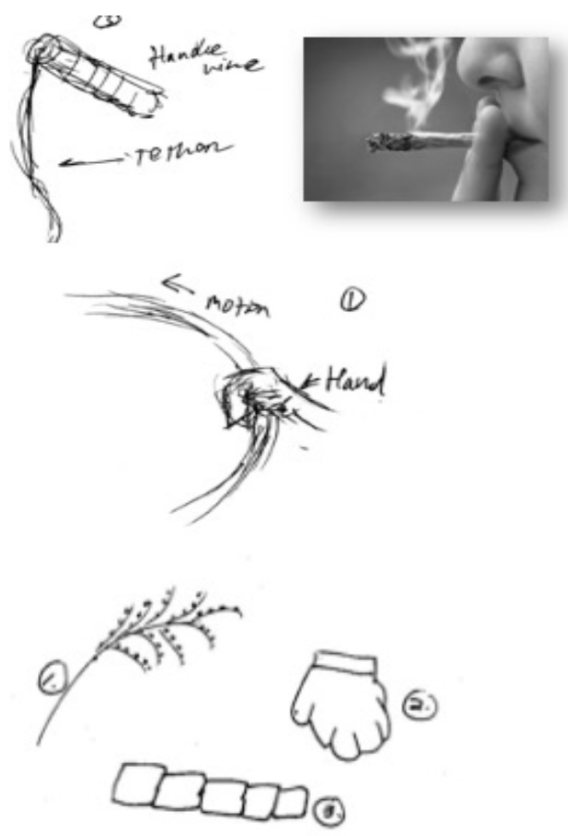


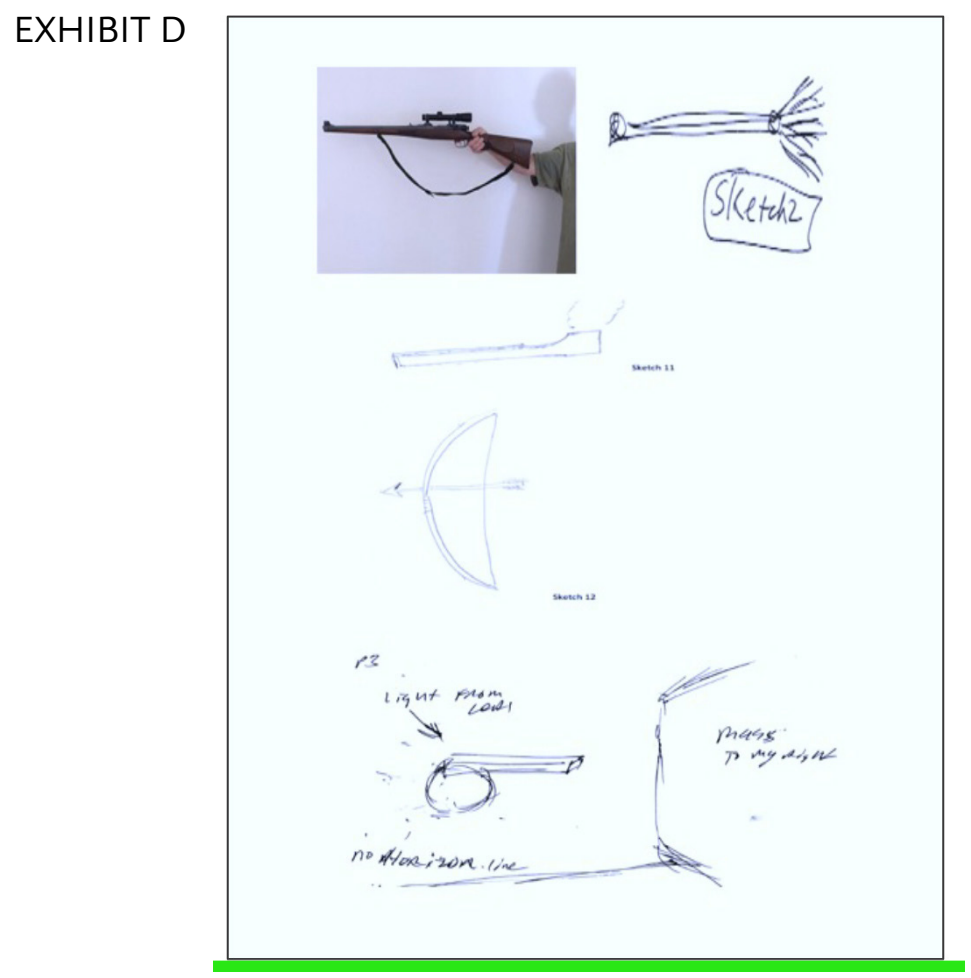

\section{EXHIBIT E}

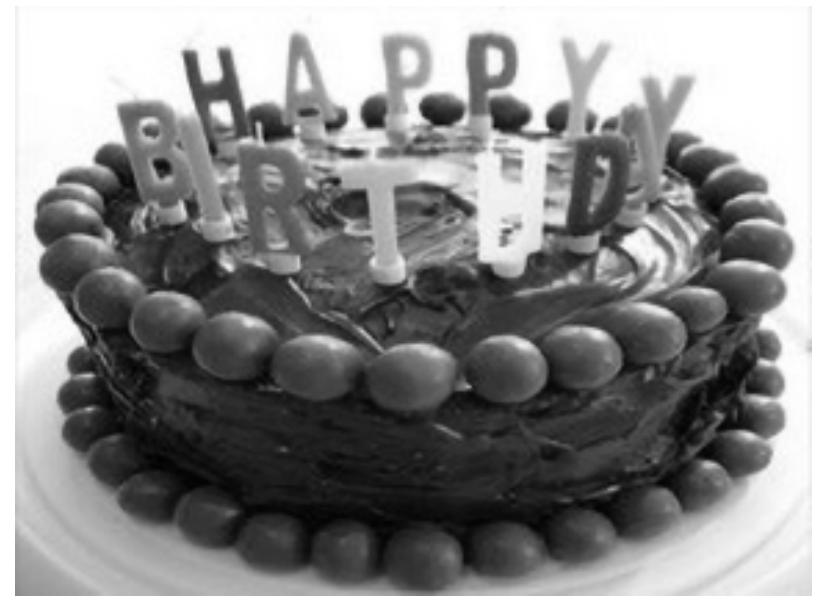

"Wedding Cake." "Feels like there is writing on it." "Many layers," "Golden Brown" 
EXHIBIT F
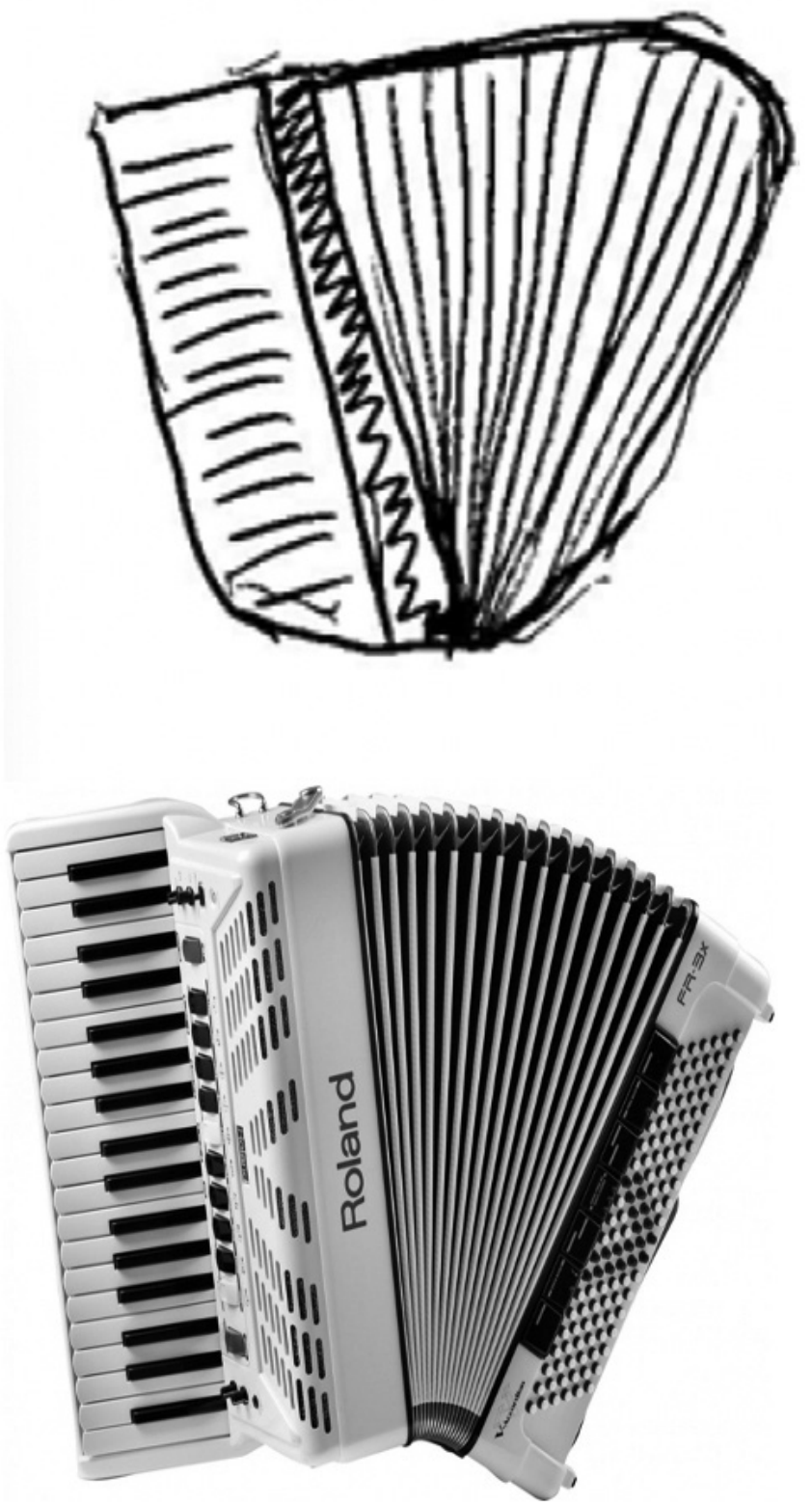

Example of potential out-of-time sequence displacement. 